\title{
Predictive value of C-reactive protein in patients treated with sunitinib for metastatic clear cell renal cell carcinoma
}

\author{
Martin Pilskog ${ }^{1,2}$, Christian Beisland ${ }^{3,5}$, Lars A. Akslen ${ }^{1,4}$, Leif Bostad ${ }^{4}$, Åse Haug ${ }^{2}$, Daniel Heinrich
} Karin M. Hjelle $e^{3,5}$ and Oddbjørn Straume ${ }^{1,2^{*}}$

\begin{abstract}
Background: Sunitinib has become mainstay first line treatment for patients with metastatic renal clear cell carcinoma (mRCC). Still, useful predictive markers of response are lacking and urgently needed for clinical decision making.

Methods: In the present study we investigated the predictive value of standard serum markers as well as clinical markers, including C-reactive protein (CRP), Neutrophil to Lymphocyte ratio (NLR) and early hypertension (eHTN) in an unselected prospective patient population treated with sunitinib for mRCC. Forty-six patients were enrolled in a prospective single-arm study of predictive markers for sunitinib response. Response rates according to RECIST 1.1 were used as primary end-point. Secondary objectives were to evaluate prognostic value of the candidate markers with regard to progression free survival (PFS) and overall survival (OS). In addition, toxicity rates and quality of life was recorded.
\end{abstract}

Results: Median PFS and OS was 9.1 months and 15.0 months, respectively. Of 38 patients evaluable for response, 1 patient had complete response (CR), 7 had partial response (PR), 18 had stable disease (SD) and 12 had progressive disease (PD). Normal CRP at baseline was significantly associated with objective response (CR + PR) $(p=0.01)$. Normal CRP was also significantly associated with improved PFS and OS (Log rank, $p=0.05$ and $<0.01$, respectively). Early hypertension, NLR and IMDC risk score were not significantly associated with response rates or survival.

Conclusion: Baseline CRP was a significant predictive factor of sunitinib response and a prognostic factor of survival. Baseline CRP might be a useful biomarker in the treatment planning of mRCC. Due to the relatively small sample size, our results need to be confirmed in larger studies.

\section{Background}

The frequent inactivation of the Von Hippel Lindau $(V H L)$ gene in clear cell renal cell carcinoma, leading to increased levels of hypoxia inducible factor 1 (HIF1) and vascular endothelial growth factor (VEGF), provides the rationale for treatment with antiangiogenic receptor tyrosine kinase (rTKI) inhibitors. Since the reporting of the first positive clinical trial [1], showing an overall survival benefit from a rTKI, sunitinib has become mainstay first line treatment for patients with metastatic renal cell

\footnotetext{
* Correspondence: Oddbjorn.straume@helse-bergen.no

${ }^{1}$ Centre for Cancer Biomarkers CCBIO, University of Bergen, Bergen, Norway

${ }^{2}$ Department of Oncology, Haukeland University Hospital, 5021 Bergen,

Norway

Full list of author information is available at the end of the article
}

carcinoma (mRCC). Although objective response rates are reported for around $50 \%$ of the patients, development of resistance to the treatment is a major problem [2]. Clearly, a subset of patients does not benefit from treatment with sunitinib, and side effects are frequent. Interestingly, hypertension is a common side effect of angiogenesis inhibitors and has been associated with improved treatment response [3]. In the research community, considerable effort has been made to identify and validate predictive biomarkers of response to sunitinib treatment, but so far, no biomarkers have been established as useful in clinical decision making and treatment planning.

There is increasing evidence to support an important role of systemic inflammation in development and progression 
of RCC [4], as recently substantiated by positive results from a clinical trial with the PD-1 inhibitor nivolumab in mRCC [5]. VEGF does not only stimulate tumor associated angiogenesis, but also plays an important role in the local immune response in wounds (physiologic) and tumors (pathologic) by inducing accumulation of immature dendritic cells, myeloid-derived suppressor cells, regulatory $\mathrm{T}$ cells, as well as by inhibiting the migration of T lymphocytes to the tumor [6]. Thus, it is relevant to also explore biomarkers primarily associated with inflammatory responses in the search for predictive markers for response to anti-VEGF therapy.

C-reactive protein (CRP) is an established biomarker for systemic inflammation, available in most clinical datasets, and provides prognostic information in several cancers including RCC [7]. Another biomarker of inflammation, neutrophil-to-lymphocyte ratio (NLR), adds prognostic information in RCC, and was recently suggested as a predictive marker of response to sunitinib in mRCC [8]. In the present trial we investigated the predictive value of serum markers, including CRP and NLR, in an unselected prospective patient population treated with sunitinib for mRCC. In addition, we report on toxicity and health related quality of life (HRQoL) data.

\section{Methods}

\section{Patients and treatment}

Between October 2007 and October 2014, a regional cohort of 77 patients with mRCC was screened for inclusion in this prospective study at Haukeland University Hospital, Bergen, Norway. Forty-six patients were enrolled after signing the informed consent sheet (CONSORT Flow Diagram, Additional file 1: Fig. S1). Inclusion criteria included: previously untreated metastatic or non-resectable clear cell RCC, WHO performance state $0-2$, no known brain metastases, evaluable tumor lesions according to RECIST (version 1.1) and no significant comorbidity or laboratory abnormalities. See Additional file 2: Table S1 for all inclusion criteria. Sunitinib was administered $50 \mathrm{mg} /$ day on schedule 4 weeks on/ two weeks off. Patients continued on treatment until disease progression, significant toxicity or consent withdrawal. Data was collected from the hospital records and included demographics, treatment modifications, adverse events, radiologic response data and survival. Data cut-off date was July 312015.

The main objective of this study was to identify and evaluate the predictive value of candidate markers readily available in a standard clinical setting, in mRCC patients treated with sunitinib. Candidate markers included early hypertension (eHTN), IMDC risk groups, baseline neutrophil to lymphocyte ratio (NLR), baseline CRP and baseline EORTC QoL symptom scale. Response rates according to RECIST 1.1 were used as primary endpoint. Secondary objectives were to evaluate prognostic value of the candidate markers with regard to progression free survival (PFS) and overall survival (OS). In addition, toxicity rates and HRQoL was recorded.

\section{Assessment of response, adverse events and quality of life}

The primary endpoint was objective response (OR) defined as complete response (CR) or partial response (PR) according to RECIST v.1.1 as well as clinical benefit (CB) defined as $\mathrm{CR}+\mathrm{PR}$ and including stable disease (SD) for more than 6 months. Disease stabilization is considered beneficial to patients experiencing progression at the time of inclusion and $\mathrm{CB}$ is frequently included as an additional statistical endpoint in trials investigating antiangiogenic drugs in which therapeutic activity and clinical benefit are present, even in the absence of radiological tumor shrinkage [9]. Importantly, all patients were in clinical and/or radiological progression at the time of inclusion. OR and CB were calculated on the basis of investigator assessment. Response evaluation by CT-scan or MRI was performed every 12 weeks. Patients with clinically evident disease progression or death due to $\mathrm{mRCC}$ before first radiological progression were recorded as progressive disease (PD). Best overall response (BOR), recorded as change in size of target lesion, was not available in these patients. PFS was defined as the time from treatment initiation until disease progression according to RECIST v.1.1. OS was defined as the time from enrollment until death of any cause.

Standard blood samples, including CRP and neutrophil/lymphocyte counts, were taken at treatment initiation and every 6 weeks during treatment. Adverse events were graded according to the National Cancer Institute Common Terminology Criteria for Adverse events, version 3.0 (CTCAE v.3.0), and were recorded at each 6-week cycle. Early hypertension (eHTN) was particularly evaluated for its potential role as a predictive marker for treatment response. We recorded eHTN in two different ways. First, we defined eHTN as either maximum post-baseline systolic blood pressure (SBP) $\geq 140 \mathrm{mmHg}$ or maximum post-baseline diastolic blood pressure (DBP) $\geq 90 \mathrm{mmHg}$ recorded at week 6 and week 12 [3]. Second, we recorded eHTN as HTN $\geq$ grade 1 defined by CTCAE v.3.0 at week 6 and week 12. All other adverse events were recorded every six weeks throughout the entire treatment period. Patients that stopped treatment due to toxicity before 1st tumor response evaluation at week 12 were not included in the analyses of response rates or PFS, but were included in the analyses of OS.

HRQoL was assessed by a validated Norwegian version of the questionnaire of the European Organization for Research and Treatment of Cancer (EORTC QLQ-C30 v.3.0) at baseline and every 12 weeks during treatment. 
The QLQ-C30 contains a global health/QoL scale, five functional scales (physical, role, cognitive, emotional, and social), three symptom scales (fatigue, pain, and nausea/vomiting) and six single items (dyspnoea, insomnia, anorexia, constipation, diarrhoea, and financial difficulties). The answers are given according to a 4-point Likert format, with the exception of questions about general health and quality of life, which are given according to a 7-point Likert format. Scores was calculated as described in the EORTC QLQ-C30 Scoring manual (3rd edition) [10]. The C 30 functional scales and the global scale were transformed so that $100 \%$ indicates best function and $0 \%$ least function of the individual QoL index, whereas the C30 symptom scales were transformed so that $0 \%$ indicates the least and $100 \%$ the most symptoms. We compared the upper quartile with the lower 3 quartiles for the symptom sum score and the lower quartile with the upper 3 quartiles for the functional sum score and global health/QoL score.

\section{Statistical analyses}

The Mann-Whitney U test was used to compare the distribution of continuous variables between two groups such as responders and non-responders. Comparisons between categorical variables were performed by using the Fisher's exact test. Spearman's rank correlation coefficient was used to test correlations between variables of interest. Logistic regression analysis was used to test the relative importance of predictive factors for sunitinib response. Cronbach's $\alpha$ was used to estimate the reliability of the global health score and the functional/symptomatic scores made up of more than one question. Kaplan-Meier estimates were applied for time-to-event endpoints such as PFS and OS, and log rank-test was applied for testing of differences between groups. Sample size calculations were based on a difference in response rate of $40 \%$ (i.e. $10 \%$ and $50 \%$ ) between groups identified by the candidate markers. Thirty-eight patients were needed to achieve a power of $80 \%$ with an $\alpha$-value of 0.05 . All $p$-values are two-sided. Statistical investigation were done using IBM SPSS Statistics version 22.

\section{Results}

\section{Patient population and treatment efficacy}

The characteristics of the 46 patients enrolled in the study are presented in Table 1 . In our cohort the median age was 63.1 (range 41.1-84.0). By July 31st, 2015 the median follow up time was 13.8 months (range 1.883.9). Twenty-six patients had prior removal of the primary tumor, twenty-four by radical and 2 by partial nephrectomy. Six patients had resection of bone metastasis, eight patients had resection of other metastasis, one patient had gamma knife radiosurgery of brain metastasis and two patients had radiation therapy against
Table 1 Baseline Patients Characteristics

\begin{tabular}{|c|c|}
\hline & Study cohort $(n=46)$ \\
\hline \multicolumn{2}{|l|}{ Age, years } \\
\hline Median & 63.1 \\
\hline Range & $41.1-84.0$ \\
\hline \multicolumn{2}{|l|}{ Sex - No. (\%) } \\
\hline Male & $29(63.0)$ \\
\hline Female & $17(37.0)$ \\
\hline \multicolumn{2}{|c|}{ WHO performance status - No. (\%) } \\
\hline 0 & $30(65.2)$ \\
\hline 1 & $16(34.8)$ \\
\hline 2 & $0(0.0)$ \\
\hline \multicolumn{2}{|l|}{ Site of metastases - No. (\%) } \\
\hline Brain & $1(2.2)$ \\
\hline Lung & $35(76.1)$ \\
\hline Pleura & $3(6.5)$ \\
\hline Liver & $4(8.7)$ \\
\hline Bone & $16(34.8)$ \\
\hline Lymph nodes & $28(60.9)$ \\
\hline \multicolumn{2}{|c|}{ Number of disease sites - No. (\%) } \\
\hline 1 & $10(21.7)$ \\
\hline 2 & $11(23.9)$ \\
\hline$\geq 3$ & $25(54.3)$ \\
\hline \multicolumn{2}{|c|}{ Hypertension before treatment - No. (\%) } \\
\hline Yes & $24(52.2)$ \\
\hline No & $22(47.8)$ \\
\hline \multicolumn{2}{|l|}{ IMDC risk score - No. (\%) } \\
\hline Good & $7(15.2)$ \\
\hline Intermediate & $16(34.8)$ \\
\hline Poor & $21(45.7)$ \\
\hline Missing & $2(4.3)$ \\
\hline \multicolumn{2}{|c|}{ Time from initial diagnosis - No. (\%) } \\
\hline$\leq 12$ months & $33(71.7)$ \\
\hline$>12$ months & $13(28.3)$ \\
\hline \multicolumn{2}{|c|}{ Prior removal of primary tumor - No. (\%) } \\
\hline Radical nephrectomy & $24(52.2)$ \\
\hline Partial nephrectomy & $2(4.3)$ \\
\hline No & $20(43.5)$ \\
\hline
\end{tabular}

Abbreviations: WHO World Health Organisation, IMDC International Metastatic Renal Cell Carcinoma Database Consortium

bone metastasis prior to sunitinib treatment. Median time on treatment was 5.7 months (range 0.5-63.0). Median time from first diagnosis of renal cell carcinoma to treatment was 3.2 months (range 0.3-124). Median time to treatment from diagnosis of metastasis was 1.4 months (range 0.3-66.5). 
By July 31 2015, median progression free survival (PFS) was 9.1 months (range 0.5-57.3) and median overall survival (OS) was 15.4 months (range 1.8-83.9). At data cut-off, 9 patients were still alive, and six patients were still on sunitinib treatment without signs of progression. Twenty-three patients started second line systemic treatment. We observed 1 complete response (CR), 7 partial responses (PR) and 18 patients had stable disease $(\mathrm{SD}) \geq 6$ months. Twelve patients showed progressive disease (PD), of which 10 were confirmed by radiology and 2 were confirmed by clinical progress before week 12. Eight patients stopped treatment before week 12 and were recorded as nonevaluable for response rates and PFS. Of these, six were due to toxicity without evidence of disease progression, one patient due to appendicitis and one protocol violation. Of interest, seven of these eight patients were females.

\section{Predictive value of pre-treatment clinical and biochemical markers and survival analyses}

The correlations between clinical, as well as biochemical markers assessed ahead of treatment initiation and sunitinib response are given in Table 2. The association between clinical, as well as biochemical markers assessed ahead of treatment initiation, PSF and OS is given in Table 3.

\section{C-reactive protein (CRP)}

Median CRP at baseline was $17.0 \mathrm{mg} / \mathrm{L}$, range $0-235 \mathrm{mg} / \mathrm{L}$. Seventeen of 37 patients evaluated for overall response had normal CRP $(\leq 10 \mathrm{mg} / \mathrm{L})$. Normal CRP at baseline was significantly associated with $\mathrm{OR}(\mathrm{CR}+\mathrm{PR})$ (Fisher's exact test, $p=0.01$ ) (Fig. 1). Seven/17 (41\%) of patients with normal CRP had an objective response to sunitinib, compared with $1 / 20$ (5\%) patients with elevated CRP had an objective response. Logistic regression analysis was used to test the relative importance of the candidate predictive factors for sunitinib response(eHTN, IMDC risk groups, baseline NLR, baseline CRP, baseline EORTC QoL symptom scale). Only CRP level at baseline was an independent predictive variable of response, with an odds ratio of $14.3(p=0.02)$ of not having an objective response if CRP was above normal $(10 \mathrm{mg} / \mathrm{L})$. CRP at baseline was significantly correlated with several other variables including age, function sum score, symptom sum score, performance status and tumor load (Additional file 3: Table S2). Median PFS was significantly longer among patients with normal CRP at baseline (median 14.7 vs 5.3 months, $\log$ rank $p=0.05$ ). Similarly, an improved OS was found in patients with normal CRP at baseline (median 26.0 vs 12.1 months, log-rank $p<0.01$ ) (Fig. 2 a, b).

\section{Neutrophil to lymphocyte ratio (NLR)}

Twenty-two of 34 (64\%) patients evaluated for response and available NLR, had NLR $\leq 3$ at baseline (median $=2.7$, range 1.0-7.9) and NLR at baseline was not significantly correlated with OR or CB. Eighty-three \% of patients evaluated for response had NLR $\leq 3$ at week 6 (median $=1.6$, range $0.4-5.9$ ) and this was not significantly correlated to OR or CB. A shift from NLR $>3$ at baseline to $\leq 3$ at week $6(n=10)$ was not significantly associated with OR or CB. Median PFS among patients with baseline NLR $\leq 3$ was significantly better than patients with baseline NLR >3 (median 14.7 vs 6.7 months, log rank $p=0.05)$. A borderline association was present between baseline NLR $\leq 3$ and OS (median 25.2 vs 13.2 months, log-rank $p=0.06$ ). High NLR at baseline was significantly correlated with increased tumor load $(r=0.43$, $p=0.005$, Spearman).

\section{Treatment induced early hypertension (eHTN)}

Applying the first definition of eHTN (SBP $\geq 140$ or DBP $\geq 90 \mathrm{mmHg}$ at week 6 and week 12) seventeen of $32 \mathrm{pa}-$ tients (53\%) evaluated for response had eHTN after week 6. Median SBP over DBP was 145 (range: 120170) $\mathrm{mmHg}$ over 89 (range: $60-170) \mathrm{mmHg}$, and was not significantly associated to OR or CB. Using the same definition at week 12, fifteen of 19 patients (79\%) had eHTN. Median SBP over DBP was 142 (range: 120-170) $\mathrm{mmHg}$ over 88 (range: $65-107) \mathrm{mmHg}$, and was significantly associated with improved $\mathrm{CB}$, but not $\mathrm{OR}$ (Fischer's exact test $p=0.04$ and $p=0.53$, respectively) (Table 2). The second definition (based on CTCAE v 3.0) of eHTN was not significantly associated OR or CB (data not presented). eHTN at week 12 was associated with improved PFS and OS (Table 3). All seven patients with increased blood pressure during the two first cycles used anti-hypertensive drug(s) at baseline.

\section{Risk scores}

The distribution of IMDC risk score [11] is given in Table 1. IMDC risk score was not significantly associated with OR or CB. Good IMDC risk score versus intermediate and poor was not significantly correlated to PFS (median 20.4 vs 9.1 vs 8.4 months, $\log$-rank $p=0.10$ ), but was significantly associated with OS (median 67.9 vs 12.7 vs 13.7 months, log-rank $p=<0.01)$. We found similar results for MSKCC risk score and WHO performance status (PS) (data not presented).

\section{Metastatic sites}

The distribution of metastatic sites is given in Table 1 . There was no significant association between metastatic site and response rates. Although present in only 3 patients, pleura metastasis was significantly associated with reduced PFS (median 2.6 vs 9.1 months, log rank 
Table 2 Univariate analyses of clinical and biochemical markers in relation to response to sunitinib

\begin{tabular}{|c|c|c|c|c|c|c|}
\hline \multirow[t]{2}{*}{ Variable } & \multicolumn{6}{|c|}{ Best overall tumor response (RECIST ver. 1.1) } \\
\hline & $\begin{array}{l}\mathrm{OR}^{1} \\
\mathrm{n}(\%)\end{array}$ & $\begin{array}{l}\mathrm{SD}^{2}+\mathrm{PD}^{3} \\
\mathrm{n}(\%)\end{array}$ & $p$ value $^{4}$ & $\begin{array}{l}C^{5} \\
n(\%)\end{array}$ & $\begin{array}{l}P D^{3} \\
n(\%)\end{array}$ & $p$ value \\
\hline Total & $8(21)$ & $30(79)$ & & 26(68) & $12(32)$ & \\
\hline Age & & & 0.69 & & & 0.73 \\
\hline$<63.1$ & $4(17)$ & 19(83) & & $15(65)$ & $8(35)$ & \\
\hline$\geq 63.1$ & $4(27)$ & $11(73)$ & & $11(73)$ & $4(27)$ & \\
\hline Sex & & & 0.17 & & & 0.45 \\
\hline Female & $4(40)$ & $6(60)$ & & $8(80)$ & 2(20) & \\
\hline Male & $4(14)$ & $24(86)$ & & $18(64)$ & 10(36) & \\
\hline Number of disease sites & & & 0.71 & & & 0.31 \\
\hline$\leq 2$ & $3(18)$ & $14(82)$ & & 10(59) & $7(41)$ & \\
\hline$>2$ & $5(24)$ & $16(76)$ & & $16(76)$ & $5(24)$ & \\
\hline Prior nephrectomy & & & 0.26 & & & 0.73 \\
\hline Yes & 6(29) & $15(71)$ & & $15(71)$ & 6(29) & \\
\hline No & $2(12)$ & $15(88)$ & & $11(65)$ & 6(35) & \\
\hline Pretreatment hypertension ${ }^{6}$ & & & 0.70 & & & 0.30 \\
\hline Yes & $5(26)$ & $14(74)$ & & $15(79)$ & $4(21)$ & \\
\hline No & $3(16)$ & $16(84)$ & & $11(58)$ & $8(42)$ & \\
\hline Treatment induced eHTN ${ }^{7} \leq$ week 6 & & & 1.00 & & & 0.70 \\
\hline Yes & $4(23)$ & $13(77)$ & & $13(76)$ & $4(24)$ & \\
\hline No & $4(27)$ & $11(73)$ & & $10(67)$ & $5(33)$ & \\
\hline Treatment induced eHTN ${ }^{8} \leq$ week 12 & & & 0.53 & & & 0.04 \\
\hline Yes & $5(33)$ & $10(67)$ & & $13(87)$ & 2(13) & \\
\hline No & $0(0)$ & $4(100)$ & & $1(25)$ & $3(75)$ & \\
\hline IMDC risk & & & 0.77 & & & 0.46 \\
\hline Good & 2(29) & $5(71)$ & & 6(86) & $1(14)$ & \\
\hline Intermediate & $3(25)$ & $9(75)$ & & $9(75)$ & $3(25)$ & \\
\hline Poor & $3(18)$ & $14(82)$ & & 10(59) & $7(41)$ & \\
\hline NLR baseline $\leq 3$ & & & 1.00 & & & 0.46 \\
\hline Yes & $5(23)$ & $17(77)$ & & $16(73)$ & $6(27)$ & \\
\hline No & $3(25)$ & $9(75)$ & & $7(58)$ & $5(42)$ & \\
\hline NLR week $6 \leq 3$ & & & 0.32 & & & 0.15 \\
\hline$\leq 3$ & $7(23)$ & $23(77)$ & & $22(73)$ & $8(27)$ & \\
\hline$>3$ & $0(0)$ & $6(100)$ & & 2(33) & $4(67)$ & \\
\hline NLR shifted from $>3$ to $\leq 3$ at week 6 & & & 1.00 & & & 0.06 \\
\hline Yes & $2(25)$ & $6(75)$ & & $6(75)$ & $2(25)$ & \\
\hline No & $0(0)$ & $3(100)$ & & $0(100)$ & $3(100)$ & \\
\hline NLR shifted from $\leq 3$ to $>3$ at week 6 & & & 1.00 & & & 0.50 \\
\hline Yes & $0(0)$ & $2(100)$ & & $1(50)$ & $1(50)$ & \\
\hline No & $5(26)$ & $14(74)$ & & $14(74)$ & $5(26)$ & \\
\hline CRP $\leq 10(\mathrm{mg} / \mathrm{L})$ & & & 0.01 & & & 0.09 \\
\hline Yes & $7(41)$ & $10(59)$ & & $14(82)$ & 3(18) & \\
\hline No & 1(5) & 19(95) & & $11(55)$ & $9(45)$ & \\
\hline
\end{tabular}


Table 2 Univariate analyses of clinical and biochemical markers in relation to response to sunitinib (Continued)

\begin{tabular}{|c|c|c|c|c|c|c|}
\hline EORTC QoL symptom scale at BL ${ }^{9}$ & & & 0.31 & & & 0.02 \\
\hline Upper quartile & $0(0)$ & $7(100)$ & & 2(29) & $5(71)$ & \\
\hline Lower 3 quartiles & $8(27)$ & $22(73)$ & & $24(80)$ & $6(20)$ & \\
\hline $\begin{array}{l}\text { Abbreviations: IMDC International Meta } \\
\text { of normal, NLR neutrophil/lymphocyte } \\
\text { 1Objective response (Complete + Parti } \\
{ }^{2} \text { Stable disease } \\
\text { 3Progressive disease } \\
{ }^{4} \text { Fisher's exact test } \\
{ }^{5} \text { Clinical benefit (OR + SD) } \\
6 \text { Defined as on anti-hypertensive treat } \\
{ }^{7} \text { Defined as systolic blood pressure(SB) } \\
{ }^{8} \text { Defined as } \geq 140 \mathrm{mmHg} \text { or diastolic b } \\
{ }^{9} \text { Quality of Life }\end{array}$ & $\begin{array}{l}\text { ell Carci } \\
\text { eactive } \\
\\
\text { nitiation } \\
\text { g or dia } \\
\text { (DBP) } \geq\end{array}$ & $\begin{array}{l}\text { base Con } \\
\text { baseline }\end{array}$ & $\mathrm{mHg} \leq$ & & & \\
\hline
\end{tabular}

$p=0.05$ ) and OS (median 10.8 vs 17.5 months, log-rank $p=0.04)$. Presence of lung metastasis was significantly associated with reduced OS (median 13.2 vs 48.2 months, $\log$-rank $p=0.04$ ). Other metastatic sites (brain, bone, liver or lymph nodes) were not significantly associated with PFS or OS.

\section{Toxicity}

Adverse events occurring during treatment with sunitinib according to CTCAE v.3.0 are summarized in Additional file 4: Table S3. The most common adverse effects of lower grade $(1+2)$ were nausea $(52.2 \%)$, anemia (47.8\%), fatigue (45.7\%) and diarrhea (39.1\%). The most common severe adverse effects (grade $3+4$ ) were hypertension (19.6\%), fatigue (15.2\%), low serum platelets (15.2\%), hand-foot skin reaction (10.9\%) and diarrhea $(10.9 \%)$. We observed one grade 5 adverse effect (death due to appendicitis) probably not related to sunitinib treatment.

\section{Health related quality of life}

The results of the HRQoL questionnaires at baseline $(n=45)$ and at the first treatment evaluation $(n=28$, after 12 weeks) are presented in Additional file 5: Table S4. Only for "Fatigue", there was a statistically significant increase in the score during treatment compared with the baseline value $(p=0.041$, Wilcoxon ranked signed test). The Cronbach- $\alpha$ of the indices derived by more than one question showed acceptable/good values (0.74-0.89), except for "cognitive function" (0.30) and "social functioning" (0.56). The Cronbach- $\alpha$ of the sum scores of functional indexes (0.80) and symptom indexes (0.79) were acceptable/good. In contrast to Global health status/QoL and Functional scale, a Symptom sum score below median was significantly associated to improved CB (Fisher's exact test, $p=0.02$, Table 2). Investigating symptom sum scores indicated that the upper quartile had significantly worse OS (median 12.7 vs 25.2 months, $\log$-rank $p=0.01$ ) and PFS (median 2.9 vs 14.7 months, $\log$-rank $p=<0.01)$. No such difference could be demonstrated for global health/QoL status or functional sum score (data not shown).

\section{Discussion}

Until recently, palliative surgery, radiation therapy and chemotherapy were the only treatment options for metastatic RCC (mRCC), and primary therapy resistance, reduced quality of life and short survival were major challenges in this patient group. Currently, three major categories of systemic treatment exist for the largest subgroup of mRCC, the clear cell carcinomas: cytokines and immune checkpoint inhibitors, anti VEGF targeted drugs and mTOR inhibitors [12]. The two latter of these new treatment options have emerged based on recent knowledge of the pathogenesis of clear cell renal cancer. The von Hippel-Lindau (VHL) tumor suppressor gene is lost or mutated in $60-90 \%$ in sporadic cases [13] and is a major contributor to development of this cancer. Loss of VHL leads to a chronic stress response state in the cells trough high levels of HIF1- $\alpha$, a transcription factor for a number of stress response proteins, including vascular endothelial growth factor (VEGF). In addition to being a potent angiogenic growth factor, VEGF plays a role in the local immune response in wounds and tumors by inducing accumulation of immature dendritic cells, myeloid-derived suppressor cells, regulatory $\mathrm{T}$ cells, as well as by inhibiting the migration of $\mathrm{T}$ lymphocytes to the tumor [6]. Renal cell cancer is regarded as highly immunogenic and angiogenic tumors, supporting VEGF as a promising target for treatment. The VEGF receptor inhibitor Sunitinib is currently first line treatment for mRCC [12], but a significant portion of the patients do not respond, and the search for good predictive markers of response has been disappointing so far. Whereas most focus in the search for predictive markers has been on angiogenesis, less focus has been on markers of immune responses. In the current study, we evaluated readily available clinical and biochemical markers, associated with systemic inflammation, for their association with response to sunitinib. 
Table 3 Survival analyses according to clinical and biochemical variables

\begin{tabular}{|c|c|c|c|c|c|c|}
\hline \multirow[t]{2}{*}{ Variable } & \multicolumn{3}{|l|}{$\mathrm{PFS}^{1}$} & \multicolumn{3}{|l|}{$O S^{2}$} \\
\hline & Median & $95 \% \mathrm{Cl}^{3}$ & $p$-value ${ }^{4}$ & Median & $95 \% \mathrm{Cl}$ & $p$-value \\
\hline$\overline{\text { Age }}$ & & & 0.29 & & & 0.47 \\
\hline$<63.1$ & 8.7 & $6.2-11.2$ & & 17.5 & $5.5-29.4$ & \\
\hline$\geq 63.1$ & 20.4 & $3.2-37.7$ & & 15.0 & $12.4-17.6$ & \\
\hline Sex & & & 0.03 & & & 0.87 \\
\hline Female & NR & - & & 12.7 & $7.3-18.2$ & \\
\hline Male & 8.7 & $6.6-10.7$ & & 18.0 & $10.5-25.4$ & \\
\hline Number of disease sites & & & 0.80 & & & 0.52 \\
\hline$\leq 2$ & 12.9 & $2.1-23.7$ & & 17.5 & $11.3-23.7$ & \\
\hline$>2$ & 9.1 & $8.3-9.8$ & & 13.9 & $10.2-17.6$ & \\
\hline Prior nephrectomy & & & 0.07 & & & $<0.01$ \\
\hline Yes & 14.7 & $5.9-23.5$ & & 26.0 & $20.1-31.8$ & \\
\hline No & 8.7 & $3.1-14.3$ & & 10.8 & $4.5-17.0$ & \\
\hline Pretreatment hypertension & & & 0.42 & & & 0.79 \\
\hline Yes & 17.0 & $6.2-27.7$ & & 18.0 & $9.4-26.5$ & \\
\hline No & 8.4 & $3.7-13.1$ & & 11.6 & $5.3-17.9$ & \\
\hline Treatment induced early hypertension ${ }^{5}$ at week 6 & & & 0.68 & & & 0.85 \\
\hline Yes & 14.7 & $9.6-19.8$ & & 18.0 & $3.5-32.5$ & \\
\hline No & 8.7 & $3.9-13.5$ & & 12.1 & $3.8-20.4$ & \\
\hline Treatment induced early hypertension ${ }^{5}$ at week 12 & & & $<0.01$ & & & $<0.01$ \\
\hline Yes & 14.7 & $10.1-19.3$ & & 26.0 & $24.1-27.9$ & \\
\hline No & 2.6 & $1.9-3.3$ & & 7.7 & $4.4-11.0$ & \\
\hline IMDC risk score & & & 0.10 & & & $<0.01$ \\
\hline Good & 20.4 & $13.1-27.7$ & & 67.9 & $38.4-97.5$ & \\
\hline Intermediate & 9.1 & $6.0-12.2$ & & 12.7 & $10.6-14.9$ & \\
\hline Poor & 8.4 & $0-17.7$ & & 13.7 & $5.4-22.1$ & \\
\hline NLR baseline $\leq 3$ & & & 0.05 & & & 0.06 \\
\hline Yes & 14.7 & $8.8-20.6$ & & 25.2 & $10.6-39.8$ & \\
\hline No & 6.7 & $2.0-11.4$ & & 13.2 & $10.3-16.1$ & \\
\hline NLR week $6 \leq 3$ & & & 0.09 & & & $<0.01$ \\
\hline Yes & 10.8 & $6.0-15.7$ & & 25.2 & $13.7-36.7$ & \\
\hline No & 1.8 & $0.2-3.5$ & & 3.8 & $3.3-4.3$ & \\
\hline NLR shifted from $>3$ to $\leq 3$ week 6 & & & $<0.01$ & & & $<0.01$ \\
\hline Yes & 8.4 & $6.0-10.7$ & & 13.2 & 7.0-19.4 & \\
\hline No & 1.3 & $0.9-1.7$ & & 3.6 & $0.8-6.4$ & \\
\hline NLR shifted from $\leq 3$ to $>3$ at week 6 & & & 0.75 & & & 0.16 \\
\hline Yes & NR & - & & 4.0 & $2.3-5.7$ & \\
\hline No & 14.7 & $9.2-20.3$ & & 26.0 & $24.2-27.7$ & \\
\hline CRP & & & 0.05 & & & $<0.01$ \\
\hline$\leq 10(\mathrm{mg} / \mathrm{L})$ & 14.7 & $2.5-26.9$ & & 26.0 & $0.6-51.4$ & \\
\hline$>10(\mathrm{mg} / \mathrm{L})$ & 5.3 & $0.9-9.8$ & & 12.1 & $8.8-15.5$ & \\
\hline EORTC QoL symptom scale at BL & & & $<0.01$ & & & 0.01 \\
\hline Upper quartile & 2.8 & $2.2-3.5$ & & 12.7 & $4.8-20.7$ & \\
\hline Lower 3 quartiles & 14.7 & $4.6-24.8$ & & 25.2 & $12.7-37.7$ & \\
\hline
\end{tabular}

Abbreviations: IMDC International Metastatic Renal Cell Carcinoma Database Consortium, LDH Lactate dehydrogenase, ULN Upper limit of normal, LLN lower limit of normal, NLR neutrophil/lymphocyte ratio, CRP C-reactive protein, NR Not reached, BL Baseline

${ }^{1}$ Progression free survival

${ }^{2}$ Overall survival

${ }^{3}$ Confidence interval

${ }^{4}$ Log rank test

${ }^{5}$ Defined as systolic blood pressure(SBP) $\geq 140 \mathrm{mmHg}$ or diastolic blood pressure (DBP) $\geq 90 \mathrm{mmHg}$ 


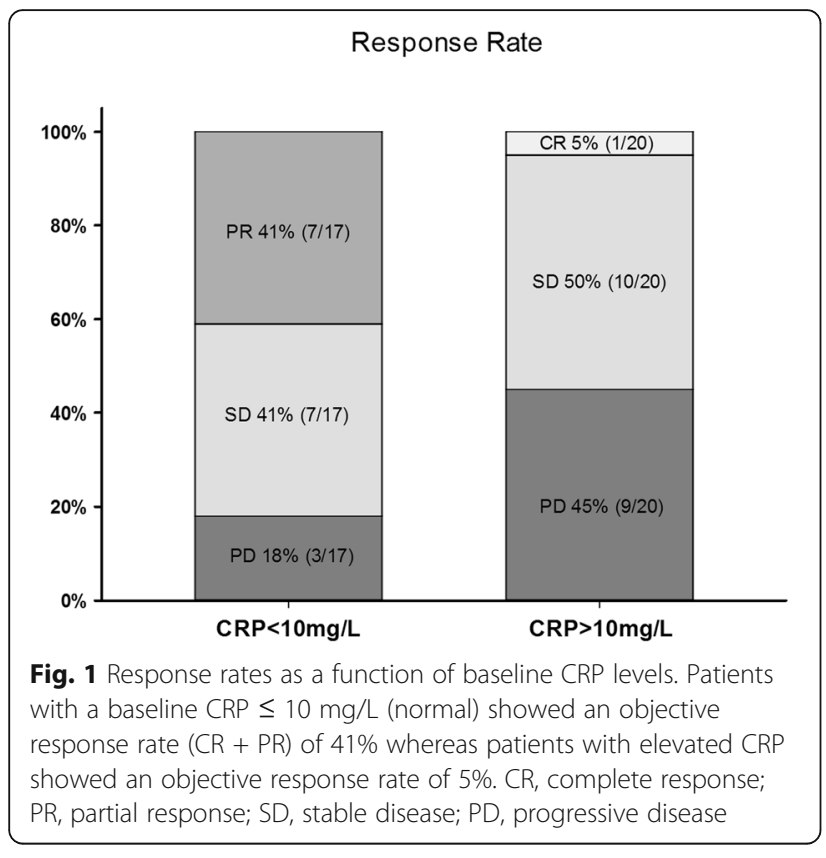

C-reactive protein (CRP) is an acute-phase protein that increases rapidly following interleukin- 6 secretion by macrophages and $\mathrm{T}$ cells following infection, inflammation and cancer [14]. CRP is a negative prognostic marker in most cancers. In the present study we found normal CRP to be a possible predictive factor of response. Whereas $41 \%$ of the patients with normal CRP at baseline experienced an objective response, this was the case for only $5 \%$ of patients with CRP levels at baseline above normal. CRP was also associated with PFS and OS supporting its role as a prognostic marker as well, and this is in line with previous reports [15-17]. Our finding supports the results of a recent study by Fujita et al. where normal level of CRP at baseline was an independent predictive marker of response by multivariate analysis [15]. In a retrospective study of 200 patients treated with sunitinib $61 \%$ of patients with normal CRP responded vs $32 \%$ of patients with elevated CRP [18]. In our trial, CRP was correlated to several factors including other markers of systemic inflammation such as high platelet counts, anemia as well as tumor load and performance status. Thus, CRP might represent a marker of disease burden identifying a patient subpopulation with poor prognosis, less likely to respond. Nevertheless, the significant association with response rates suggests that CRP might be a useful marker, in addition to other clinical and biochemical features to consider prior to initiation of systemic treatment. IL-6 is an important tumor-promoting protein associated with stress responses, inflammation and angiogenesis [19]. Through its major downstream target STAT3 several tumor promoting pathways are activated, including HIF1- $\alpha$ and VEGF [19]. Moreover, IL6 have direct stimulating effect on endothelial cells, and has been implicated in resistance to antiVEGF therapy [20]. Being closely correlated to IL6 expression, increased CRP levels might therefore be a surrogate marker of IL6 driven disease, again being associated with expression of multiple angiogenic factors [21], thus less responsive to specific anti-VEGF treatment like sunitinib. Our results indicate that an inflammatory response, defined by high CRP is associated with poor response to sunitinib and poor prognosis in these patients. The effect of sunitinib on inhibiting the angiogenesis supporting and immunosuppressive effect of VEGF, thus seem to be more pronounced in patients with a non-inflammatory state defined by normal CRP.

The neutrophil-to-lymphocyte ratio (NLR) is also a marker of systemic inflammation in cancer patients and was found to add prognostic [22] and predictive [8] information in RCC in retrospective studies. Like CRP, NLR is readily available in standard blood samples in a regular clinical setting. Our NLR counts were comparable to what has been reported in other clinical datasets.
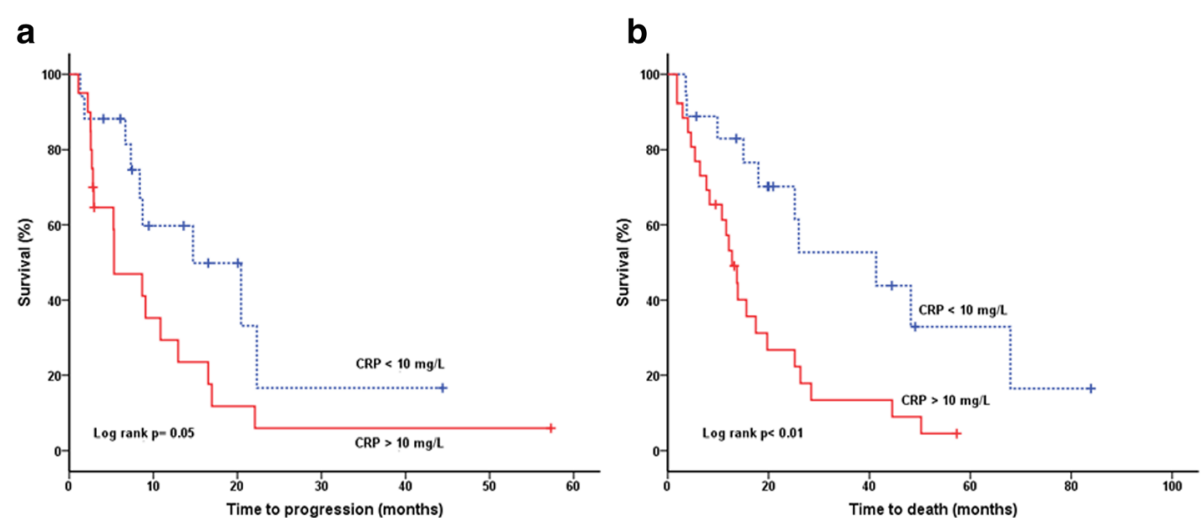

Fig. 2 Kaplan-meier estimates of a) progression free survival (PFS) and b) overall survival (OS) grouped by CRP level. The normal CRP ( $\leq 10 \mathrm{mg} / \mathrm{L}$ ) cohort showed significantly improved PFS and OS compared with patients with elevated CRP 
Although significantly associated with CRP, we did not find a statistically significant association with sunitinib response or survival. The significant correlation with performance status and tumor load suggests that NLR is a nonspecific marker of disease burden. Still, due to relatively small sample size and low statistical power, our data must be interpreted carefully.

Treatment induced early hypertension (eHTN) was not significantly associated with treatment response in our dataset. In our patient population, the baseline blood pressure was slightly higher when compared with the clinical trial population studied by Rini et al. [3], and $52 \%$ of our patients were hypertensive at baseline. Still, the number of patients recorded as having sunitinib induced eHTN after cycle 1 and 2, using the same criteria, was nearly the same ( $80 \%)$. eHTN at week 12 was associated with improved survival, but this is most likely due to the fact that the responders in the study stayed on treatment long enough to develop hypertension. Even if pharmacodynamically interesting, as eHTN occurs after sunitinib initiation it is not going to be an applicable predictive marker in the clinic.

Our finding that higher baseline HRQoL symptoms score is prognostic for PFS and OS in treatment with $\mathrm{Su}-$ nitinib is in line with the earlier report by Cella et al. [23]. In that report, however, a different QoL tool was used. Herrmann et al. demonstrated by using EORTC QLQC30, that "global QoL" was prognostic for PFS [24]. Our study did not confirm this finding. In general, there were only small changes in HRQoL scores form baseline to 12 weeks. Herrmann et al. also showed a relatively small change in the different HRQoL scales after 12 weeks [24]. This could be due to the administration of Sunitinib (4 weeks on/ 2 weeks off), with subsequent remission of eventual treatment induced symptoms. There are indications that long-term survivors might retain a good HRQoL over years, as described by Carmichael et al. [25].

A major challenge in studies exploring predictive markers of treatment response in clinical data-sets is the fact that most of the candidate predictive markers are prognostic as well, thus significantly correlated with PFS and OS independent of the treatment given. Combined predictive and prognostic markers are best evaluated in two-arm trials. In single arm trials such as ours, response rates according to RECIST are superior to PFS and OS as primary end-point when assessing predictive markers of treatment response. Many biomarker studies in mRCC have been performed retrospectively in data-sets from large clinical trials, and these patients are frequently positively selected and do not optimally reflect the normal patient population. The strength of our study is the prospective design and the "Real-World" patient population enrolled, reflecting a normal clinical setting. When compared with large retrospective multicenter studies as well as smaller single-center studies, the majority of our patients were in the poor risk group according to IMDC criteria. Whereas the portion of poor-risk patients varies between 18 and $33 \%$ in comparable studies [8, 11, 15], $46 \%$ of our patients belonged to this group. In addition, all patients were in confirmed clinical and/or radiological progression at the time of inclusion. Accordingly, patients with very slow progression or stable metastases were observed without systemic treatment and screened for inclusion in the study only after confirmed disease progression. In comparison with clinical phase III trials, PFS and OS were lower in our patients. Compared with the adverse events reported in clinical trials [26], the frequency of toxicity from sunitinib in metastatic renal cell cancer recorded in our study was similar, or somewhat less frequent. Especially, the hematological toxicity including anemia, neutropenia, thrombocytopenia and lymphopenia was less frequent in our trial, although using the same criteria (CTCAE v. 3.0). The most likely explanation for this discrepancy is that we assessed adverse events, including laboratory, every 6 weeks, where most of the patients were off the drug in the $4+2$ weeks cycle.

In addition to the lack of a control group, our study has some weaknesses. First, the number of patients included is low and thereby the study lacks the significant power to detect minor differences in response rates between groups based on the biomarkers under investigation. Thus, our finding should be validated in an independent and larger cohort of patients. Second, CRP and NLR are non-specific markers of inflammation and angiogenesis, and further studies are required to identify the key regulators controlling the systemic responses to metastatic disease. In this report, we focused on biomarkers available in standard clinical blood samples routinely used in the clinic. Further studies of candidate biomarkers in serum and plasma, such as IL6 and IL8 are ongoing.

\section{Conclusion}

In conclusion, in this prospective study of sunitinib in patients with $\mathrm{mRCC}$ we found that normal level of sCRP at baseline is significantly associated with improved response rates and might serve as guidance in the selection of optimal treatment. Still, due to the relatively small sample size and low statistical power, our results need to be confirmed in larger studies.

\section{Additional files}

Additional file 1: Fig. S1. CONSORT 2010 Flow Diagram. (DOCX 36 kb)

Additional file 2: Table S1. Inclusion criteria. (DOCX $17 \mathrm{~kb})$

Additional file 3: Table S2. Correlations between CRP and other variables. (DOCX $15 \mathrm{~kb}$ )

Additional file 4: Table S3. Adverse effects. (DOCX $20 \mathrm{~kb}$ )

Additional file 5: Table S4. Summary of quality of life (QoL) scores. (DOCX $14 \mathrm{~kb}$ ) 


\section{Abbreviations}

IMDC: International Metastatic Renal Cell Carcinoma Database Consortium; MSKCC: Memorial Sloan Kettering Cancer Center

\section{Funding}

The study was supported by grants from The Norwegian Research Council, The Norwegian Cancer Society as well an unrestricted grant from Pfizer Norway. The authors alone are responsible for the content and writing of the paper. This work was partly supported by the Research Council of Norway through its Centres of Excellence funding scheme, project number 223250

\section{Availability of data and materials}

All participants in the study signed an informed consent sheet providing the investigators permission to create a clinical dataset. According to the approval from the local ethical committee as well as from the hospital data protection board, the clinical dataset cannot be made publicly available.

\section{Authors' contributions}

MP, CB, LAA, OS: Conception and design, acquisition of data, analysis and interpretation of data, project funding, and were major contributors in writing the manuscript. LB performed the histological examination of the $H \& E$ stained tumor biopsies, and was a major contributor in writing the manuscript. ÅH, DH and KMH collected the clinical data including the QoL data, and were major contributors in writing the manuscript. All authors have read and approved the final version of the manuscript.

\section{Ethics approval and consent to participate}

The study was conducted in accordance with the ethical principles of the Declaration of Helsinki and the International Conference on Harmonization of Good Clinical Practice. The protocol was approved by the Regional Ethics Committee (REK number 080/07) and the Norwegian Medicines Agency. All participating patients provided signed informed consent before enrolment.

\section{Consent for publication}

Not applicable

\section{Competing interests}

The authors declare that they have no competing interests.

\section{Publisher's Note}

Springer Nature remains neutral with regard to jurisdictional claims in published maps and institutional affiliations.

\section{Author details}

${ }^{1}$ Centre for Cancer Biomarkers CCBIO, University of Bergen, Bergen, Norway. 2Department of Oncology, Haukeland University Hospital, 5021 Bergen, Norway. ${ }^{3}$ Department of Urology, Haukeland University Hospital, 5021 Bergen, Norway. ${ }^{4}$ Department of Pathology, Haukeland University Hospital, 5021 Bergen, Norway. ${ }^{5}$ Department of Clinical Medicine, University of Bergen, Bergen, Norway. ${ }^{6}$ Department of Oncology, Akershus University Hospital, 1478 Lørenskog, Norway.

Received: 3 January 2017 Accepted: 28 August 2017

Published online: 31 August 2017

\section{References}

1. Motzer RJ, Hutson TE, Tomczak P, Michaelson MD, Bukowski RM, Oudard S, Negrier S, Szczylik C, Pili R, Bjarnason GA, et al. Overall survival and updated results for sunitinib compared with interferon alfa in patients with metastatic renal cell carcinoma. J Clin Oncol. 2009;27(22):3584-90.

2. Rini $\mathrm{Bl}$, Atkins MB. Resistance to targeted therapy in renal-cell carcinoma. Lancet Oncol. 2009;10(10):992-1000

3. Rini BI, Cohen DP, DR L, Chen I, Hariharan S, Gore ME, Figlin RA, Baum MS, Motzer RJ. Hypertension as a biomarker of efficacy in patients with metastatic renal cell carcinoma treated with sunitinib. J Natl Cancer Inst. 2011;103(9):763-73.

4. Mantovani A, Allavena P, Sica A, Balkwill F. Cancer-related inflammation. Nature. 2008:454(7203):436-44.

5. Motzer RJ, Escudier B, McDermott DF, George S, Hammers HJ, Srinivas S, Tykodi SS, Sosman JA, Procopio G, Plimack ER, et al. Nivolumab versus Everolimus in advanced renal-cell carcinoma. N Engl J Med. 2015;
6. Voron T, Marcheteau E, Pernot S, Colussi O, Tartour E, Taieb J, Terme M. Control of the immune response by pro-angiogenic factors. Front Oncol. 2014;4:70.

7. Casamassima A, Picciariello M, Quaranta M, Berardino R, Ranieri C, Paradiso A, Lorusso V, Guida M. C-reactive protein: a biomarker of survival in patients with metastatic renal cell carcinoma treated with subcutaneous interleukin2 based immunotherapy. J Urol. 2005;173(1):52-5.

8. Keizman D, Ish-Shalom M, Huang P, Eisenberger MA, Pili R, Hammers $H$, Carducci MA. The association of pre-treatment neutrophil to lymphocyte ratio with response rate, progression free survival and overall survival of patients treated with sunitinib for metastatic renal cell carcinoma. Eur J Cancer. 2012;48(2):202-8.

9. Ratain MJ, Eckhardt SG. Phase II studies of modern drugs directed against new targets: if you are fazed, too, then resist RECIST. J Clin Oncol. 2004:22(22):4442-5.

10. Fayers P, Aaronson N, Bjordal K, Groenvold M, Curran D, Bottomley A, Group obotEQoL: The EORTC QLQ-C30 scoring manual (3rd edition). In. Brussels: the European organisation for research and treatment of Cancer; 2001.

11. Heng DY, Xie W, Regan MM, Harshman LC, Bjarnason GA, Vaishampayan UN, Mackenzie M, Wood L, Donskov F, Tan MH, et al. External validation and comparison with other models of the international metastatic renal-cell carcinoma database consortium prognostic model: a population-based study. Lancet Oncol. 2013;14(2):141-8.

12. Jonasch E, Gao J, Rathmell WK. Renal cell carcinoma. BMJ. 2014;349:94797.

13. Kaelin WG Jr. The von Hippel-Lindau tumor suppressor protein and clear cell renal carcinoma. Clin Cancer Res. 2007;13(2 Pt 2):680s-4s.

14. Ljungberg B, Grankvist K, Rasmuson T. Serum interleukin-6 in relation to acute-phase reactants and survival in patients with renal cell carcinoma. Eur J Cancer. 1997:33(11):1794-8.

15. Fujita T, Iwamura M, Ishii D, Tabata K, Matsumoto K, Yoshida K, Baba S. Creactive protein as a prognostic marker for advanced renal cell carcinoma treated with sunitinib. International journal of urology : official journal of the Japanese Urological Association. 2012;19(10):908-13.

16. Kawai Y, Osawa T, Kobayashi K, Inoue R, Yamamoto Y, Matsumoto H, Nagao K, Hara T, Sakano S, Nagamori S, et al. Factors prognostic for survival in Japanese patients treated with Sunitinib as first-line therapy for metastatic clear cell renal cell cancer. Asian Pacific journal of cancer prevention: APJCP. 2015;16(14):5687-90.

17. Teishima J, Kobatake K, Kitano H, Nagamatsu H, Sadahide K, Hieda K, Shinmei S, Shoji K, Inoue S, Hayashi T, et al. The impact of change in serum C-reactive protein level on the prediction of effects of molecular targeted therapy in patients with metastatic renal cell carcinoma. BJU Int. 2016; 117(6B):E67-74.

18. Beuselinck B, Vano YA, Oudard S, Wolter P, De Smet R, Depoorter L, Teghom C, Karadimou A, Zucman-Rossi J, Debruyne PR, et al. Prognostic impact of baseline serum $\mathrm{C}$-reactive protein in patients with metastatic renal cell carcinoma (RCC) treated with sunitinib. BJU Int. 2014:114(1):81-9.

19. Middleton K, Jones J, Lwin Z, Coward J. Interleukin-6: an angiogenic target in solid tumours. Crit Rev Oncol Hematol. 2014:89(1):129-39.

20. Kwon KA, Kim SH, SY O, Lee S, Han JY, Kim KH, Goh RY, Choi HJ, Park KJ, Roh MS, et al. Clinical significance of preoperative serum vascular endothelial growth factor, interleukin-6, and C-reactive protein level in colorectal cancer. BMC Cancer. 2010;10:203.

21. Gopinathan G, Milagre C, Pearce OM, Reynolds LE, Hodivala-Dilke K, Leinster DA, Zhong H, Hollingsworth RE, Thompson R, Whiteford JR, et al. Interleukin-6 stimulates defective angiogenesis. Cancer Res. 2015; 75(15):3098-107.

22. Ohno Y, Nakashima J, Ohori M, Hatano T, Tachibana M. Pretreatment neutrophil-to-lymphocyte ratio as an independent predictor of recurrence in patients with nonmetastatic renal cell carcinoma. J Urol. 2010;184(3):873-8.

23. Cella D, Bushmakin AG, Cappelleri JC, Charbonneau C, Michaelson MD, Motzer RJ. Baseline quality of life as a prognostic survival tool in patients receiving sunitinib for metastatic renal cell carcinoma. Br J Cancer. 2012; 106(4):646-50.

24. Herrmann E, Gerss J, Bierer S, Kopke T, Bolenz C, Hertle L, Wulfing C. Pretreatment global quality of health predicts progression free survival in metastatic kidney cancer patients treated with sorafenib or sunitinib. J Cancer Res Clin Oncol. 2009;135(1):61-7. 
25. Carmichael C, Yuh BE, Sun V, Lau C, Hsu J, Saikia J, Liu X, Wilson T, Ferrell B, Pal SK. Quality of life in patients with metastatic renal cell carcinoma: assessment of long-term survivors. Clin Genitourin Cancer. 2013:11(2):149-54.

26. Motzer RJ, Hutson TE, Cella D, Reeves J, Hawkins R, Guo J, Nathan P, Staehler M, de Souza P, Merchan JR, et al. Pazopanib versus sunitinib in metastatic renal-cell carcinoma. N Engl J Med. 2013;369(8):722-31.

Submit your next manuscript to BioMed Central and we will help you at every step:

- We accept pre-submission inquiries

- Our selector tool helps you to find the most relevant journal

- We provide round the clock customer support

- Convenient online submission

- Thorough peer review

- Inclusion in PubMed and all major indexing services

- Maximum visibility for your research

Submit your manuscript at www.biomedcentral.com/submit 